\title{
Proper Generalized Decomposition Applied on a Rotating Electrical Machine
}

\author{
L. Montier ${ }^{1,2}$, T. Henneron ${ }^{1}$, S. Clénet ${ }^{1}$ and B. Goursaud ${ }^{2}$ \\ ${ }^{1}$ Univ. Lille, Centrale Lille, Arts et Métiers Paris Tech, HEI, EA 2697 - L2EP -Laboratoire \\ d'Electrotechnique et d'Electronique de Puissance, F-59000 Lille, France \\ 2 EDF R\&D, ERMES, 7 Boulevard Gaspard Monge, 91120 Palaiseau, France
}

\begin{abstract}
The Proper Generalized Decomposition (PGD) is a model order reduction method which allows to reduce the computational time of a numerical problem by seeking for a separated representation of the solution. The PGD has been already applied to study an electrical machine but at standstill without accounting the motion of the rotor. In this paper, we propose a method to account for the rotation in the PGD approach in order to build an efficient metamodel of an electrical machine. Then, the machine metamodel will be coupled to its electrical and mechanical environment in order to obtain accurate results with an acceptable computational time on a full simulation.
\end{abstract}

Index Terms_Finite Element Method, Model Order Reduction, Proper Generalized Decomposition, Electrical Machine, Metamodel

\section{INTRODUCTION}

A PPLYING the Finite Element (FE) method to model electrical machines is now common. This approach enables to obtain accurate results but requires solving large scale systems, leading to a high computational cost. Moreover, when the equations of the model depend on a significant amount of physical parameters, the required number of solutions of the FE model to precisely characterize the problem explodes. In order to reduce the computational cost, model order reduction methods have been proposed. The two most common model order reduction methods which deal with parametric problems are the Reduced Basis (RB) and the Proper Generalized Decomposition (PGD). The RB approach consists in approximating the solution in a reduced basis, leading to a numerical problem with few unknowns. As for the PGD, the solution is approximated with a separated representation, allowing to efficiently deal with parametric problems [1]. The PGD has already been successfully applied to model electric devices such as 3-phase transformers [2]. However, the PGD approach has not been used to model problems accounting for the motion of an electrical machine.

The approximation of the FE solution, proposed by the PGD method, is very fast to evaluate. Thus, the PGD approach seems to be very interesting in order to build a metamodel of an electric device. With this approach, it is then possible to couple the device to its electrical or mechanical environment in order to simulate the behavior of an electrical machine over its full operating range. In this paper, we apply the PGD approach to a $2 \mathrm{D}$ linear FE model of a rotating electrical machine. Generally, the ratio between the mechanical and electrical time constants are often more than one order of magnitude. Then, the time step is necessarily of one order less than the electrical time constant whereas the period of simulation is generally of one order greater than the mechanical time

Manuscript received December 1, 2012; revised August 26, 2015. Corresponding author: L. Montier (email: laurent.montier@ensam.eu). constant. For this kind of simulation where the number of time steps can be large, it can be of great interest to replace the full FE model by a metamodel. The metamodel is then used to study the electrical machine in its mechanical and electrical environment. A comparison with the full model is made in terms of time computation and accuracy.

\section{FE MAGNETOSTATIC PROBLEM OF AN ELECTRICAL} MACHINE

Let us consider a magnetostatic problem of a $2 \mathrm{D}$ synchronous machine in a domain $D$ with boundary $\Gamma$. The device is composed of a rotor domain $D_{\theta}$ and a stator one $D \backslash D_{\theta}$. Four stranded inductors supplied by the currents $i_{j}, j=0, \ldots, 3$ are considered as shown in Fig.1. The linear magnetostatic vector potential formulation is given by:

$$
\operatorname{curl}\left(\mu(\boldsymbol{x})^{-1} \operatorname{curl} \boldsymbol{A}(\boldsymbol{x})\right)=\sum_{j=0}^{3} i_{j} \boldsymbol{N}_{j}(\boldsymbol{x})
$$

with $\boldsymbol{A}$ the vector potential. $\boldsymbol{N}_{j}$ is the unit current density vector flowing through the $j^{\text {th }}$ stranded winding and $i_{j}$ its associated current. $\mu(\boldsymbol{x})$ denotes the magnetic permeability of the linear isotropic materials, which is equal to $\mu$ in the ferromagnetic material and $\mu_{0}$ otherwise. Moreover, the following boundary condition is applied on $\Gamma:\left.(\boldsymbol{A} \times \boldsymbol{n})\right|_{\Gamma}=0$. The FE model is obtained by approximating $\boldsymbol{A}$ with $N_{x}$ linear nodal functions in 2D. Furthermore, the Overlapping Finite Element Method is used in order to take into account the motion of the rotor without any remeshing process [3]. Finally, the linear system of equations describing our problem reads:

$$
\left(\boldsymbol{M}(\mu)+\boldsymbol{M}_{O v l}(\theta)\right) \boldsymbol{X}=\sum_{j=0}^{3} \boldsymbol{F}_{j} i_{j}
$$

with $\boldsymbol{X}$ the unknown vector in $\mathbb{R}^{N_{x}}$ whose $k^{\text {th }}$ component is the value of $\boldsymbol{A}$ on the $k^{t h}$ node. $\boldsymbol{M}(\mu)$ is the stiffness square matrix of size $N_{x} \times N_{x}$ which is symmetric positive semi-definite while $M_{O v l}(\theta)$ denotes the overlapping matrix 
accounting for the motion of the rotor after a rotation of angle $\theta$. As for $\boldsymbol{F}_{j}, j=0, \ldots, 3$, they refer to the four vectors of size $N_{x}$ depending on the unitary current density $\boldsymbol{N}_{j}$. Then, the solution of the FE equation is denoted by $\boldsymbol{X}\left(\theta, \mu, i_{0}, i_{1}, i_{2}, i_{3}\right)$.

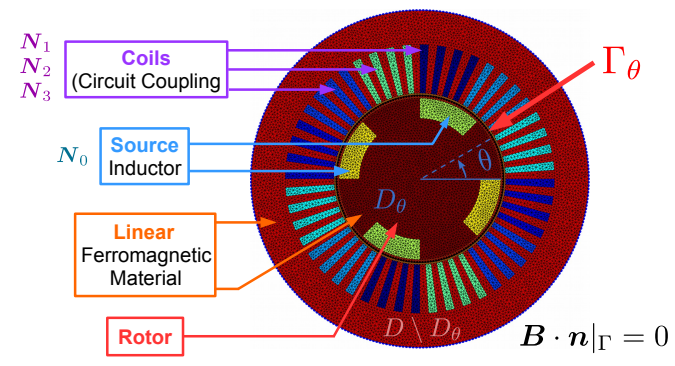

Fig. 1. Electrical machine

\section{METAMOdEL OF THE SYNCHRONOUS MACHINE WITH THE PROPER GENERALIZED DECOMPOSITION}

In this section, a metamodel of the synchronous machine is constructed by applying the PGD approach. This method allows to build an approximation $\boldsymbol{Y}\left(\theta, \mu, i_{0}, i_{1}, i_{2}, i_{3}\right)$ of the solution $\boldsymbol{X}\left(\theta, \mu, i_{0}, i_{1}, i_{2}, i_{3}\right)$ which is much faster to evaluate.

\section{A. Decomposition of the metamodels}

Before applying the PGD on the FE problem (2), one has to take advantage of the linearity with respect to the right hand size of this equation. Indeed, the FE solution can be decomposed as:

$$
\boldsymbol{X}\left(\theta, \mu, i_{0}, i_{1}, i_{2}, i_{3}\right)=\sum_{k=0}^{3} i_{k} \boldsymbol{X}_{k}(\theta, \mu)
$$

where $\boldsymbol{X}_{k}(\theta, \mu)$ is solution of the following FE problem:

$$
\left(\boldsymbol{M}(\mu)+\boldsymbol{M}_{\text {Ovl }}(\theta)\right) \boldsymbol{X}=\boldsymbol{F}_{k} .
$$

This system of equations is derived from (2) with $i_{j}=\delta_{j}^{k}, j=$ $0 \ldots 3$ and $\delta_{j}^{k}$ denoting the kronecker delta.

Thus, our approach consists in computing a metamodel $\boldsymbol{Y}_{k}(\theta, \mu)$ approximating $\boldsymbol{X}_{k}(\theta, \mu)$ for each $k$ in $\{0, \ldots, 3\}$, in order to reconstruct later on the full metamodel as in (3):

$$
\boldsymbol{Y}\left(\theta, \mu, i_{0}, i_{1}, i_{2}, i_{3}\right)=\sum_{k=0}^{3} i_{k} \boldsymbol{Y}_{k}(\theta, \mu)
$$

\section{B. Proper Generalized Decomposition}

PGD consists in looking for an approximation $\boldsymbol{Y}_{k}(\theta, \mu)$ of the parametric solution $\boldsymbol{X}_{k}(\theta, \mu)$ in a separated representation. With this type of approximation, $\boldsymbol{Y}_{k}(\theta, \mu)$ is written as a sum of products of functions which depend on a single parameter (FE discretization, $\theta$, or $\mu$ ). At the $m^{t h}$ iteration of the algorithm, the PGD approximation reads:

$$
\begin{aligned}
\boldsymbol{Y}_{k}^{m}(\theta, \mu) & =\sum_{j=1}^{m} \Psi_{x}^{j} \Psi_{\theta}^{j}(\theta) \Psi_{\mu}^{j}(\mu) \\
& =\boldsymbol{Y}_{k}^{m-1}(\theta, \mu)+\Psi_{x}^{m} \Psi_{\theta}^{m}(\theta) \Psi_{\mu}^{m}(\mu)
\end{aligned}
$$

where $\boldsymbol{Y}_{k}^{m-1}(\theta, \mu)$ has been computed at the previous PGD iteration. Thus, the only unknowns to determine are at this step $\Psi_{x}^{m} \in \mathbb{R}^{N_{x}}, \Psi_{\theta}^{m}(\theta)$ and $\Psi_{\mu}^{m}(\mu)$ (two scalar functions). Parameter $k$ has been removed in these three functions for sake of simplicity. To do this, the first step is to express the residual vector $\boldsymbol{R}_{k}^{m}(\mu, \theta)$ of equation (4) arising from the PGD approximation:

$$
\boldsymbol{R}_{k}^{m}(\mu, \theta)=\left(\boldsymbol{M}(\mu)+\boldsymbol{M}_{O v l}(\theta)\right) \boldsymbol{Y}_{k}^{m}(\mu, \theta)-\boldsymbol{F}_{k}
$$

Once $\Psi_{\theta}^{m}(\theta)$ and $\Psi_{\mu}^{m}(\mu)$ have been initialized to a given value, $\Psi_{x}^{m}$ is computed by solving the following equation:

$$
\left\langle\boldsymbol{R}_{k}^{m}(\mu, \theta), \Psi_{\theta}^{m}(\theta) \Psi_{\mu}^{m}(\mu)\right\rangle=0
$$

where $\langle.,$.$\rangle denotes the appropriate scalar product. In this case,$ it is computed over the angular and permeability spaces by using the trapezoidal rule (piecewise linear approximation). For the FE space, the scalar product is $\boldsymbol{L}^{2}(\boldsymbol{D})$. The equation (9) actually consists in projecting the residual $\boldsymbol{R}_{k}^{m}(\mu, \theta)$ (defined on the spatial, angular and permeability dimensions) onto the angular and permeability dimensions. This leads to a spatial equation (equivalent to a FE problem). Moreover, the unknown vector $\boldsymbol{\Psi}_{x}^{m}$ in (9) is hidden in $\boldsymbol{R}_{k}^{m}(\mu, \theta)$ according to equation (8).

In the same way $\Psi_{\theta}^{m}(\theta)$ is the solution of the following equation where the residual has been projected onto the spatial and permeability dimension:

$$
\left\langle\boldsymbol{R}_{k}^{m}(\mu, \theta), \Psi_{x}^{m} \Psi_{\mu}^{m}(\mu)\right\rangle=0
$$

As for $\Psi_{\mu}^{m}(\mu)$, it is solution to:

$$
\left\langle\boldsymbol{R}_{k}^{m}(\mu, \theta), \boldsymbol{\Psi}_{x}^{m} \Psi_{\theta}^{m}(\theta)\right\rangle=0
$$

Finally, these three coupled equations are solved with a fixed point approach until convergence is reached. Then, $m$ is incremented if the prescribed accuracy of the metamodel is not reached. Fig. 2 summarizes the PGD algorithm, where $\eta=\left\|\Delta \boldsymbol{\Psi}_{x}^{m}\right\|+\left\|\Delta \Psi_{\theta}(\theta)^{m}\right\|+\left\|\Delta \Psi_{\mu}(\mu)^{m}\right\|$ is the fixed point error indicator and $\epsilon=\left\|\boldsymbol{Y}^{m}-\boldsymbol{Y}^{m-1}\right\|$ is the PGD error indicator. These norms come from the scalar products previously defined.

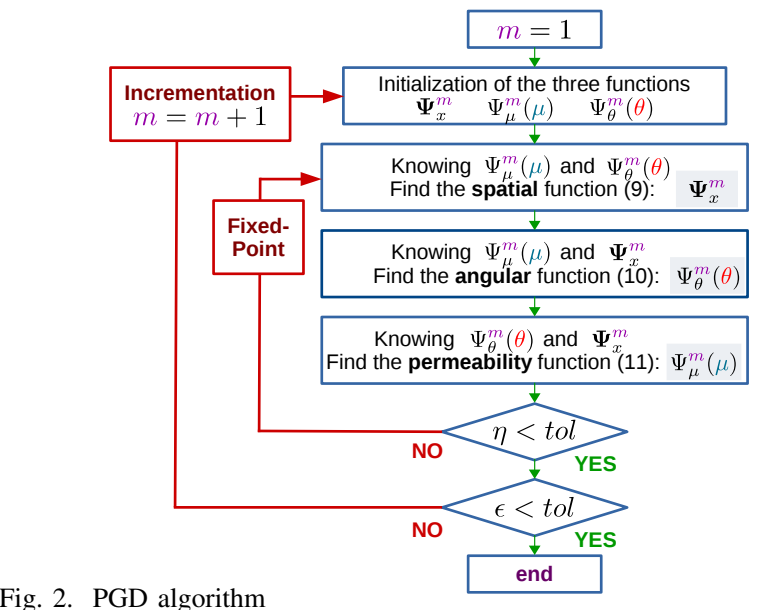

Fig. 2. PGD algorithm 


\section{Piecewise affine decomposition of the Operators}

In order to compute the scalar products with the residual vector very efficiently in (9), (10) and (11), the equations on which the PGD is applied should be written under a piecewise affine decomposition (PAD). This means that the main operator $\left(M(\mu)+M_{O v l}(\theta)\right)$ is written as a sum of products of operators, which only depend on a single parameter [4]. For instance, the PAD of a given operator $O$ that depends on $n$ parameters is: $O\left(p_{1}, \ldots, p_{n}\right)=\sum_{q=1}^{M} O_{1}^{q}\left(p_{1}\right) \cdot O_{2}^{q}\left(p_{2}\right) \ldots O_{n}^{q}\left(p_{n}\right)$, with $O_{i}\left(p_{i}\right)$ operators which only depend on $p_{i}, \forall i \in[1, \ldots, n]$.

In the following, we will show how we deal with this constraint linked to a PAD.

\section{1) Spatial/permeability operator $M(\mu)$}

Dealing with this problem for $\boldsymbol{M}(\mu)$ is trivial since this operator has an underlying PAD. Indeed, by introducing $M_{f m}$ and $\boldsymbol{M}_{\text {air }}$, the curl-curl matrices respectively accounting for the materials in the ferromagnetic materials and for the air/coils, one can simply write:

$$
\boldsymbol{M}(\mu)=\mu_{0} \boldsymbol{M}_{\text {air }}+\mu \boldsymbol{M}_{f m}
$$

\section{2) Spatial/angular operator $\boldsymbol{M}_{O v L}(\theta)$}

For this operator, the problem is more complicated since $M_{O v l}(\theta)$ has not an underlying PAD. To find an approximation of this operator in an appropriate decomposition, the idea is to precompute $\boldsymbol{M}_{O v l}(\theta)$ for $N_{\theta}$ given values $\theta_{N_{\theta}}$ in $[0,2 \pi]: M_{O v l}^{k}=M_{O v l}((k-1) \Delta \theta), k=$ $1, \ldots, N_{\theta}$ with $\Delta \theta=2 \pi / N_{\theta}$. Then, the overlapping operator is approximated in the following PAD:

$$
\boldsymbol{M}_{O v l}(\theta) \approx \sum_{k=1}^{N_{\theta}} \boldsymbol{M}_{O v l}^{k} \alpha^{k}(\theta)
$$

with $\alpha^{k}(\theta)=1$ if $\theta \in[(k-1) \Delta \theta, k \Delta \theta[$ and is null elsewhere. Remark 1: Even though the angular grid is quite fine, computing the overlapping matrices $M_{O v l}^{k}$ is cheap in terms of time and memory space since they are sparse matrices restricted to nodes located on the two sides of the overlapping region (a ring in the airgap in our case).

Remark 2: We can mention that we had met some convergence issues to construct directly the metamodel $\boldsymbol{Y}$ without decomposing it into 4 submetamodels $\boldsymbol{Y}_{k}$, with $k=1, \ldots, 4$. Indeed, the fixed point method did not converge for each iteration, leading to an inaccurate PGD model. This can be explained by the fact that the separable representation imposed by the PGD (see [2]) is not suited for problems on which the superposition principle can be applied.

Now that the PGD metamodel has been presented, the coupling of the synchronous machine with its environment is studied.

\section{Coupling the PGD metamodel of the SYNCHRONOUS MACHINE WITH ITS ENVIRONMENT}

In the section, the coupling of the PGD metamodel with the mechanical and electrical environment is now presented. For instance, one may be interested in simulating an electrical generator with some profile of the driving torque. Moreover, taking into account the electrical environment of the machine can be done through solving circuit equations or by coupling the metamodel with an electrical network software such as EMTP-RV [5].

\section{A. Electrical environment}

In order to take into account the electrical environment, circuit equations can be coupled to the PGD metamodel. In our case, they are:

$$
\frac{\mathrm{d} \phi_{j}(\boldsymbol{Y})}{\mathrm{d} t}+L_{j} \frac{\mathrm{d} i_{j}(t)}{\mathrm{d} t}+R_{j} i_{j}(t)=0, \quad j=\{1,2,3\}
$$

where $L_{j}$ and $R_{j}$ respectively denote the inductance and the resistor associated to the $j^{\text {th }}$ winding of the stator. $\phi_{j}(\boldsymbol{Y})$ is the magnetic flux flowing through the $j^{t h}$ winding which is expressed as (see (6) for the definition of $\boldsymbol{Y}$ and $\boldsymbol{Y}_{i}$ ):

$\phi_{j}(\boldsymbol{Y})=\boldsymbol{F}_{j}^{t} \boldsymbol{Y}_{0} i_{0}(t)+\boldsymbol{F}_{j}^{t} \boldsymbol{Y}_{1} i_{1}(t)+\boldsymbol{F}_{j}^{t} \boldsymbol{Y}_{2} i_{2}(t)+\boldsymbol{F}_{j}^{t} \boldsymbol{Y}_{3} i_{3}(t)$

Using this definition of the magnetic flux, equation (14) can be solved in the time domain with a Backward Euler scheme.

\section{B. Mechanical environment}

The mechanical behaviour of the machine can be modelled through the following mechanical equation:

$$
J \frac{\mathrm{d}^{2} \theta}{\mathrm{d} t^{2}}+f \frac{\mathrm{d} \theta}{\mathrm{d} t}=\Gamma_{E M}(\boldsymbol{Y})-\Gamma_{M e c h}
$$

where $J$ is the inertial momentum of the rotor, $f$ a friction constant and $\Gamma_{M e c h}$ the driving torque. $\Gamma_{E M}$ is the electromagnetic torque which can be expressed through the virtual work principle as [6]:

$$
\Gamma_{E M}(\boldsymbol{Y})=\boldsymbol{Y}^{t} \boldsymbol{K} \boldsymbol{Y}
$$

where $\boldsymbol{K}$ is a sparse squared matrix of size $N_{x} \times N_{x}$. Equation (16) can be solved using an explicit scheme and by chaining electrical and mechanical equations [7]. This approach makes sense since the time constant of the mechanical equation is much larger than the one of the electrical equations for synchronous machines.

Remark 3: $\boldsymbol{K}$ is assembled for $q$ nodes located on a close band elements on the airgap. This means that $\boldsymbol{K}$ lines are null if the related nodes are not in this band. Thus, operation (17) is not $O\left(N_{x}\right)$ but $O(q)$ and can be efficiently performed online.

Remark 4: Compared to some other metamodeling approaches based on equivalent circuit where the lumped parameters are extracted from specific FE simulations, the PGD approach enables to reconstruct easily the field distribution. This is very interesting for computing the field distribution in the electrical machine for any parameter value.

\section{Validation of the PGD metamodel}

The $2 \mathrm{D}$ mesh of the electrical machine is presented in Fig. 1 (17248 elements and 8913 nodes). The angular grid is discretized on $N_{\theta}=288$ points in $[0,2 \pi]$, and the permeability grid on $N_{\mu}=100$ uniformly distributed points in 
$\left[10^{2} \mu_{0}, 10^{4} \mu_{0}\right]$. Figure 3 shows the currents flowing through the 3 windings of the stator with $i_{0}=1$ on a full mechanical period in a short circuit simulation. The currents are computed with both the FE code and the PGD approximation, using Matlab direct solver on equidistant time steps. The waveforms obtained with the PGD match the one from the FE code: This good accuracy is obtained with $m=80$ PGD modes for each metamodel $\boldsymbol{Y}_{k}^{80}, k=0, \ldots, 3$. Figure 4 presents the torque computed from the same simulation. One can see a good agreement with the FE simulation, even though the error is clearly bigger for this quantity. Indeed, the torque is a quadratic quantity computed through the virtual work principle, by deriving the nodal linear functions along the rotation angle $\theta$. This leads to an amplification of the approximation error due to PGD method, which explains why the error is bigger on this term.

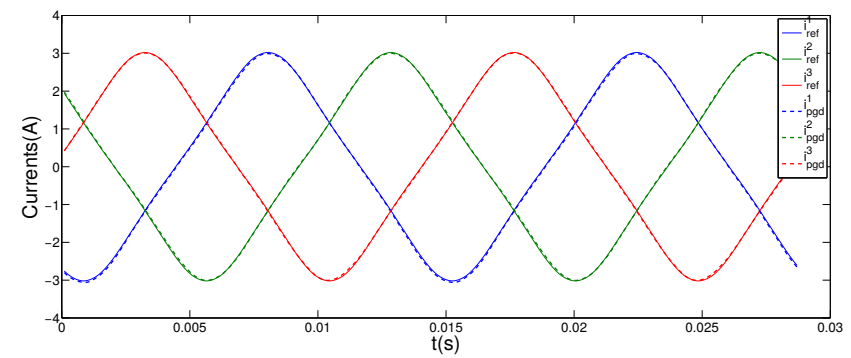

Fig. 3. Currents in the stator inductors

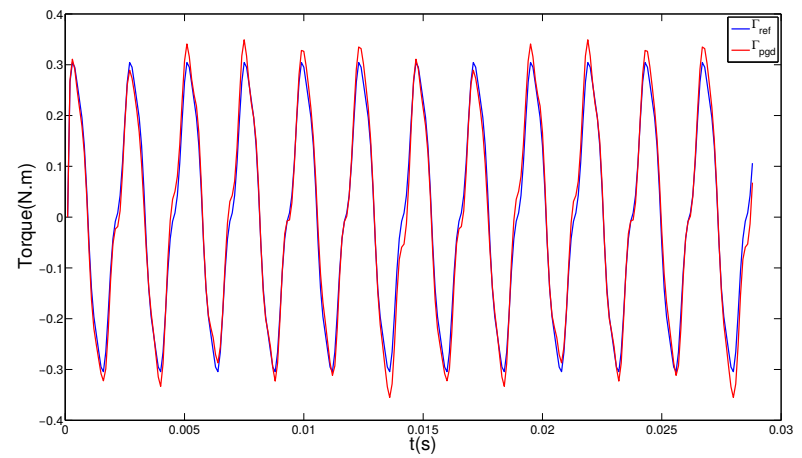

Fig. 4. Elecromagnetic torque

\section{Simulation of the PGD metamodel coupled with its} environment

Finally, a simulation of the start-up of a synchronous generator is performed using the PGD metamodel for $N_{t}=$ $10^{6}$ time steps accounting for $T=100 \mathrm{~s}\left(\Delta T=10^{-4} \mathrm{~s}\right)$. The resistors and inductances on the stator phases are set to $R_{j}=200 \Omega$ and $L_{j}=0.1 H$ for $j=1,2,3$. The direct current is set to $i_{0}=1 A$. The driving torque $\Gamma_{M e c h}$ imposed to the rotor and the rotation speed profile during the simulation are shown in Fig. 5. As for Fig. 6, it shows the currents flowing through the three phases of the stator.

Table 1 gives both the computational complexity and time of the PGD and the FE model, with $n_{f p}$ denoting the maximum number of iterations of the fixed-point approach in the PGD algorithm (see Fig. 2). In this table, the linear systems are assumed to be solved with sparse LU. Moreover, the " $3+q$ " in the Online complexity is due to the fact that only the three fluxes and the values along the $q$ nodes in the airgap need to be interpolated for the FE equation. The online speedup obtained with the PGD is about 900. However, one must temper this result because the FE model is still rather small and no symmetries of the machine were taken into account.
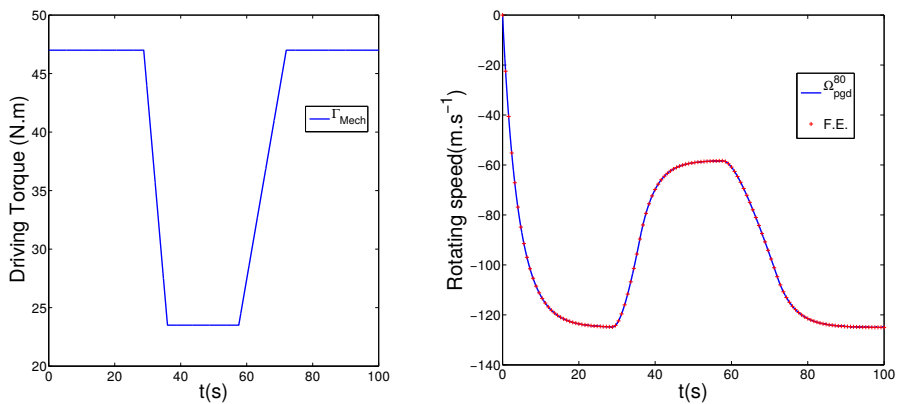

Fig. 5. Driving torque and rotating speed of the rotor vs the time

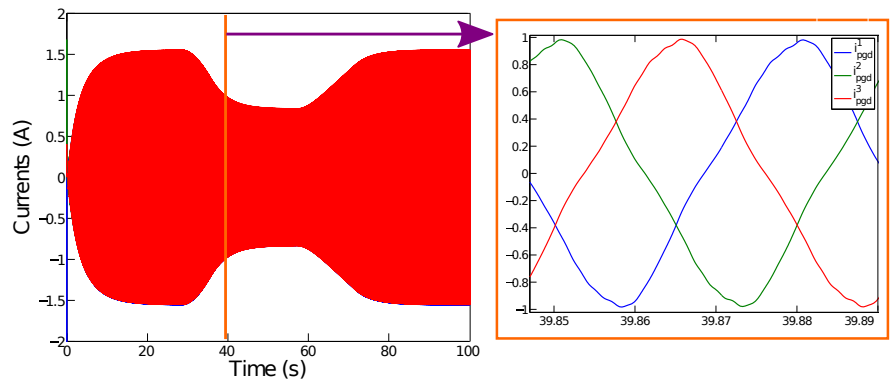

Fig. 6. Currents in the stator phases vs the time

TABLE I

COMPUTATIONAL EFFORTS

\begin{tabular}{|c|c|c|}
\hline & Complexitiy & Time(s) \\
\hline FE model & $\mathcal{O}\left(N_{t} N_{x}^{2}\right)$ & 60963 \\
\hline PGD construction (Offline) & $\mathcal{O}\left(m n_{f p}\left(N_{x}^{2}+N_{\theta}^{2}+N_{\mu}^{2}\right)\right)$ & $3038 s$ \\
\hline PGD model (Online) & $\mathcal{O}\left(N_{t} m\left(3+q+N_{\theta}+N_{\mu}\right)\right)$ & $65 s$ \\
\hline
\end{tabular}

\section{CONCLUSION}

A metamodel of a PGD synchronous machine has been proposed in this paper. It allows to reach a speedup of about 900 compared to a FE code while keeping accurate results. These two points make this approach quite promising in order to be coupled with an electrical network software such as EMTP-RV [5].

\section{REFERENCES}

[1] Chinesta, F and Ammar, A and Leygue, A and Keunings, Roland. An overview of the proper generalized decomposition with applications in computational rheology. Journal of Non-Newtonian Fluid Mechanics, 166(11):578-592, 2011.

[2] Thomas Henneron and Stephane Clenet. Application of the PGD and DEIM to solve a 3D Non-Linear Magnetostatic Problem coupled with the Circuit Equations. Magnetics, IEEE Transactions, PP(99), 2015.

[3] Tsukerman, I. A. Overlapping finite elements for problems with movement. IEEE Transactions on Magnetics, 28(5):2247-2249, 1992.

[4] Alessandra Bernardi, Jerome Brachat, Pierre Comon, and Bernard Mourrain. General tensor decomposition, moment matrices and applications. Journal of Symbolic Computation, 52:51-71, 2013.

[5] http://emtp-software.com. EMTP-RV.

[6] Ren, $\mathrm{Z}$ and Razek, A. Local force computation in deformable bodies using edge elements. Magnetics, IEEE Transactions on, 28(2):1212-1215, 1992.

[7] Laurent Montier, Thomas Henneron, Stéphane Clénet, and Benjamin Goursaud. Transient simulation of an electrical rotating machine achieved through model order reduction. Advanced Modeling and Simulation in Engineering Sciences, 3(1):1, 2016. 